Casos clínicos.

\title{
ABSCESO SUB-DURAL
}

\author{
Por el Dr. MARIANO LATORRE B. \\ Asesor Neuróogo. \\ Hospital Luis Calyo Mackenna. Cátedra dz Padiatria del Prof. A. Ariztia.
}

Nos ha movido a presentar este caso su escasa frecuencla en nuestro medio y porque durante su permanencia en $\mathrm{el}$ Servicio se discutieron muchas hipótesis para explicar la etiologia de su hipertensión intra craneana.

En la literatura nacional sólo existe un trabajo anterior publicado en los Archivos de la Sociedad de Cirujanos de Hospital por Poblete y Valladares y cuya casuistica alcanza a 14 casos, todos ellos en adultos.

El absceso sub-dural reconoce como causa las infecciones del oído, senos para nasales o faringe $y$ la osteomielitis del cráneo. Con frecuencia la infección alcanza por continuidad a través del tegmen timpani, pared posterior de los senos frontales, techo del etmoides o huesos de la bóveda, produciendo primero un absceso extra-dural. La infección de aquí, a través de los vasos de la duramadre, alcanza la membrana interna, causando el absceso, o más propiamente, el empiema sub-dural. En otras ocasiones la infección se hace a través de las conexiones venosas existentes entre las estructuras ya nombradas y la duramadre. En estos casos no hay absceso extra-dural previo. Otro tipo de infección por continuidad se hace a traves de los vasos tributarios de los senos, especialmente el longitudinal, en casos de tromboflebitis de éstos.

Establecido el empiema sub-dural, puede tomar dos cursos clínicos. Fulminante, caso en el cual la evolución es extraordinariamente rápida y en la cual el pronóstico es muy grave, de no maediar la intervención inmediata. En un $40 \%$ la infección toma un curso crónico, formando una membrana aislante y permitiendo el desarrollo de síntomas de hipertensión intra craneana y de lesiones focales corticales.

Sintomatología: Aunque ocasionalmente la sintomatología suele ser muy escasa, en la mayoría existe una gran variedad de sintomas.

Entre los síntomas generales se destacan ligera leucocitosis de 15 a 30,000 con muy poca temperatura y una ligera elevación del pulso. Entre los síntomas que se refieren al sistema nervioso, sin duda los más prominentes son la cefalea y el rómito, que traducen la hipertensión intra craneana. Le siguen, en orden de frecuencia, el estupor, delirio y ocasionalmente el coma. E1 edema de papila se describe como muy poco frecuente. El líquido céfalo-raquídeo ofrece con frecuencia un aspecto opalescente, con ligero aumento de los leucocitos, globulinas ligeramente positivas $\mathrm{y}$ el resto normal.

Desde el punto de vista focal es donde la sintomatología adquiere su caracterís. tica. Ya que el exudado ocupa una gran extensión dentro del espacio sub-dural, los sintomas focales cerebrales son variables y fluctuantes, pudiendo el enfermo presentar paresias de las extremidades contralaterales, convulsiones Jacksonianas, afasia nominal o motora, he- 
rianopsia, paresia del VI par, parálisis de los movimientos oculares conjugados. Cuando esta sintomatologia es por presión sobre las estructuras corticales adyacentes, los sintomas son transitorios, pero cuando ya se ha producido supuración focal encefálica, algunos de ellos se transforman en permanentes.

Desde el punto de vista diagnóstico existe gran dificultad para diferenciar el absceso cerebral del empiema subdural. En gran número de casos el điagnóstico es más bien quirúrgico. En los casos de empiema sub-dural fulminante puede ser muy difícil su diferenciación con la meningitis aguda purulenta. En estos casos la presencia de sintomas focales y la ausencia de gérmenes en el líquido con glucosa que permanece normal son factores decisivos.

Desde el punto de vista pronóstico la mayoría de las estadísticas revisadas dan una mortalidad que asciende hasta el $65 \%$. Todos están de acuerdo en manifestar que esta alta mortalidad se debe a la dificultad diagnóstica, ya que un tratamiento quirúrgico oportuno mejora notablemente el pronóstico.

El tratamiento es necesariamente quirúrgico.

CASO CLINICO: Lactante de 1 año 10 meses, cuya enfermedad se inicia el 15 de abril de 1951 en forma brusca, con temperatura a]ta, que alcanza a $39: 5$, decaimiento, vómitos. El cuadro febril, sin osellaclones, se mantiene durante 15 dias. El compromiso del estata general fué en alomento, presentando fosteriormente a la fase aguda, cefaleas como sintoma predominante. Dos meses antes de su ingreso notan aumento de volumen nodular con un trayecto ístujoso en la mej1lla derecha.

Ingresa al servicio el 6 de mayo de 1951.

Antecedentes familiares y personales sin importancla.

A su ingreso los exámenes clíntco y neurológlco revelan: activo, lủcido. Estado nutritivo deficiente. Gran apatía. Nódulo induravo de más o monos $1 \mathrm{~cm}$, sobresaliente, en la mejilla derecha y que por expresión deja fluir pequeña cantidad de pus espeso amarillento. Aumento consfderable del cráneo $(59 \mathrm{~cm})$ en relación con el tórax $(49 \mathrm{~cm})$. No hay separaclón de suturas ni stgno de Macewen. Fontanela anterior de $1 \times 1$. Gran edema de paplla. Tonus muscular muy disminuido. Deam- bulación sólo con apoyo. Marcha algo taloneada, con gran ampliación de la base. Pruebas cerebelosas no se pudieron practicar por falta de colaboración del paciente. Reflejos profundos simétricos algo disminuidos.

Con esta apreciación clínica se orlenta el estudio hacia un proceso expansivo intracraneano, probablemente de fosa posterior.

Los examenes de rutina practicados arrojan resultados normales. Solo llama la atencion una sedimentación muy elevada, de 131 $\mathrm{mm} y$ tuberculmas intensemente positivas. Aun iuancio la radograría de torax lué normal, se pranteo la hupotesis de un tuperculoma. En esta curcunstancia se sospechó en una puerta de entrada extrapulmonar, culpandose al noduio indurado de la mejilla derecha, que segun la bistoria tenía una evolucion torplda de 2 meses. Se practicó biopsia de esta zona sin encontrar tejido especínico. Una radogratía osea de esta reglón permitió apreciar un foco de osteitis del matar derecho.

Ia radtografia de crâneo demastró las signos clasicos de hlpertensión intracraneana. Planteada la hipótesis diagnóstica se solicitó la colaboración del Instituto Central de Neurocirugia, en donde, al practicar la punctón para ventriculografia, caen en el lado derecho en un absceso, del cual se extraen 5-6 $\mathrm{cm}$ de pus, cuyo cultivo fué negativo. Se deja Nosylan $y$ se delimita un absceso de 5-6 cm de largo por 3-4 de profundidad y a 3-4 col de la línea media. Se dejó penlctina en la cavidad, con lo cual se obtuvo mejoria del paciente. Controles ventrículográficos reallzados posteriormente daban solamente la pisualización del sistema ventricular izquierdo, a pesar de dejársele en posiclón para inyectar el sistema ventrlcular a derecha. Se decidió, con el fin de determinar la forma $y$ posición del ventrículo al lado derecho, efectuar una ventriculografía en las sitios clásicos para la trepano punción occipital, comprobándose la presencia, a $4 \mathrm{~cm}$ de profundidad, de una gruesa cápsula que no se atraviesa por la aguja de Cushing. Al retirar la aguja salió pus, aparentemente del espacio sub-dural. Se decidió explorar por vía frontal, volviendo a encontrar, a la misma profundidad, la cápsula antedicha. Se decide entonces efectuar la creneotomia exploradora con la sospecha de un absceso sub-dural. Efectuada ésta, se comprueba un absceso sub-dural de más o menos $2 \mathrm{~cm}$ de cavidad con una gruesa cápsula interna y externa. Se efectua una limpieza quirúrgica lo mas prolija pasible y se extraen muestras para su estudio histológico.

Desde esta operación se notó una franca mejoria del paciente, tanto desde el punto de vista psíquico como del estado general. Gana más o menos 1 kllo en 25 dias. 
Comentario: En este niño llamó la atención desde su ingreso la discordancia entre la sintomatología hipertensiva y la ausencia de síntomas focales.

Justamente la correcta avaluación de este hecho clínico debió hacer sospechar un proceso de las envolturas sin compromiso de la substancia cerebral. De no mediar el hallazgo casual durante las maniobras para la ventriculografía, no habría habido un diagnóstico correcto $\mathrm{y}$, por lo tanto, las posibilidades quirúrgicas y el pronóstico muy desfavorables.

Resumen: Se analiza brevemente la etiopatogenia del empiema sub-dural. Se llama la atención sobre la dificultad en el diagnóstico oportuno y se presenta un caso clínico. En él, por. exclusión, y como hallazgo operatorio durante las maniobras para practicar ventriculografía, se hizo el diagnóstico, permitiendo al enfermo una recuperación total: Llamó la atención en el curso clínico de disparidad entre la gran sintomatologia hipertensiva intracraneana y la ausencia de sintomas focales cerebrales.

\section{Summary.}

The etiology and pathogenesis of subdural empyema are briefly reviewed. The diagnostic difficulties encountered and the clinical history of a 22 months old infant with this condition are described. In this case, while performing a ventriculography the diagnosis was made. During the course of the disease the dysparity between the great hypertensive intracraneal symptomatology and the absence of focal cerebral symptoms was noticeable.

\section{Bibliografía.}

BRUNNER, HANS, - Intracranial complications of Ear Nose and Throat. Year-Book. Publisher. 1946.

COURVHLE, C. B. - Subdural Empyema secondary to purulent Frontal Sinusitis. Archives of otolaringology. March 1944.

KUBHK, c. A. and ADAMS, R. D. - Subdural Empyema. Brain. Vol. 66: 1-88, march. 1943.

POBLETE y VALLADARES. - Absceso subdural. Archiqos de Cirujanos de Hospital. Sept. 1950 . 